\title{
Noisy quantum Parrondo games
}

\author{
David A. Meyer* \\ Project in Geometry and Physics, Department of Mathematics \\ University of California/San Diego, La Jolla, CA 92093-0112
}

\begin{abstract}
Alternating two fair coin flipping games can create a winning game. Such a Parrondo game is a discrete model for a thermal ratchet. Recently we have constructed quantum versions of these coin flipping games that display the same "paradoxical" behavior. In this paper we add noise to these quantum Parrondo games in order that they can be compared with continuum models of quantum ratchets. Simulation of these models reproduces one of the most interesting features of quantum ratchets: current inversion.
\end{abstract}

Keywords: Quantum ratchet, quantum lattice gas automata, quantum random walk, decoherence

\section{INTRODUCTION}

Parrondo's game is a classical coin flipping game constructed by alternating two losing games ${ }^{1,2}$. For careful parameter choices the resulting game is a winning game. Motivated by the origin of Parrondo's game as a discrete model for thermal ratchets $^{1,2}$, we have shown that it can be understood as a random walk on a one dimensional lattice - a one particle probabilistic lattice gas automaton (LGA) - with a spaceand time-dependent bias $^{3}$. A natural question from each point of view- games $^{4}$, $\operatorname{ratchets}^{5,6}$, and random walks $/ \mathrm{LGA}^{7}$ - is the existence of a quantum version of Parrondo's game. We have answered this question positively: A quantum random walk - a one particle quantum lattice gas automaton (QLGA) - with a spaceand time-dependent potential can display essentially the same apparently paradoxical behavior as does Parrondo's game ${ }^{3}$.

This behavior can be seen most clearly in the quantum setting in the case of alternating fair games that combine to produce a winning game ${ }^{8}$. In fact, this is the case that is closest to the motivating phenomenonratcheting. Ratcheting, in either the classical or quantum setting, is a nonzero current created by applying an unbiased evolution rule at some timesteps and a locally biased evolution rule at others, where the locally biased rule is globally unbiased - if it is applied at every timestep no current is created. As we will review in $\S 2$ and $\S 3$, we have also shown that a quantum random walk/QLGA with appropriate potentials models a quantum ratchet $^{8}$.

One motivation for studying ratcheting is that it occurs in real systems, both natural and artificial ${ }^{9}$. This means, of course, that a discrete model of a perfectly unitary quantum ratchet is less applicable than a discrete model of a quantum ratchet subject to decoherence, i.e., noise. Our goal in this paper is to construct such a model - a noisy quantum Parrondo game; we do so in $\S 4$.

A priori it is not obvious that this can be done in a way that preserves any remnant of the current created by quantum correlations; measurement of the quantum system at every timestep (complete decoherence), for example, would not. Continuous models for quantum ratchets ${ }^{5,6}$ seem to offer little guidance - the approximation methods used there do not apply to our discrete model, nor do they fit very well within the modern point of view of quantum computing ${ }^{10}$ which partly motivates our interest in these questions. Nevertheless, as we show in $\S 5$, noise can be added to quantum Parrondo games in a way that reproduces

* dmeyer@math.ucsd.edu 
one of the most interesting phenomena of quantum ratchets: current inversion. We summarize and outline some future directions in $\S 6$.

\section{PARRONDO GAMES AS RANDOM WALKS}

In Parrondo games a gambler flips one of several coins, depending on his current capital. When he wins, his capital increases by 1; when he loses, it decreases by 1 . The simple case of Parrondo's "paradox" that corresponds to thermal ratcheting is exemplified as follows: Let game $A$ be a flip of an unbiased coin, so that $\operatorname{Pr}($ win $)=\frac{1}{2}$. Let game $B$ consist of two biased coins - a negatively biased one (with $\operatorname{Pr}($ win $\left.)=\frac{1}{10}\right)$ that is flipped when the gambler's capital is a multiple of 3 and a positively biased one (with $\operatorname{Pr}($ win $)=\frac{3}{4}$ ) that is flipped otherwise.

Repeatedly playing $A$ is clearly a fair gamethe expected change in capital is 0 after any number of iterations. Repeatedly playing $B$ is also a fair game ${ }^{2,3}$, in the sense that the expected winnings are asymptotically constant, neither increasing nor decreasing with the number of iterations. Alternating plays of these games in the sequence $A A B B$, repeated, or even in random sequence, however, produce a winning game: the expected change in capital is positive and approximately proportional to the number of plays. Figure 1 shows the expected results for an initial capital of 0 and 100 plays, in each of these cases.

Integer-valued capital corresponds to a lattice discretization of the position of a Brownian particle modelling diffusion in a one-dimensional potential. Game $A$ models a constant potentialthe random walk is locally unbiased - and game $B$

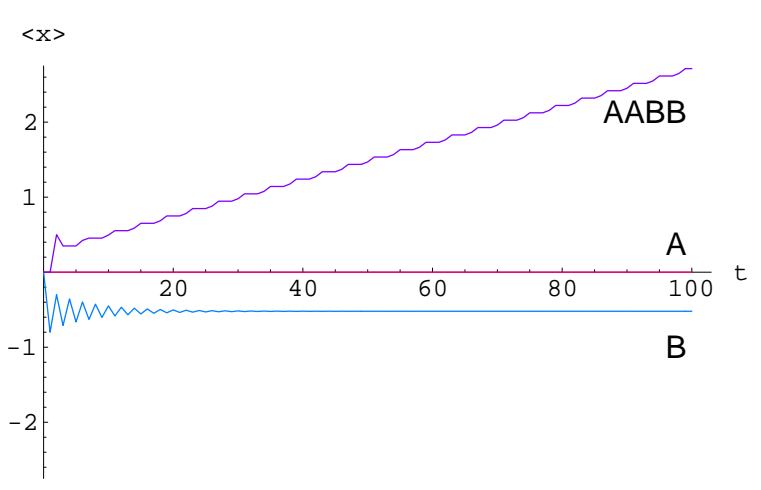

Figure 1. The expected payoffs for games $B, A$ and $A A B B$ as a function of number of plays $t$. Although $A$ and $B$ are fair games, the combination $A A B B$ is a (strongly) winning game.

models a sawtooth potential - the bias is periodic in space, sharply negative at every third lattice site and moderately positive at the sites in between, but globally the random walk is unbiased - asymptotically there is no current. Alternating the games mimics a "flashing ratchet" that drives the particle to the right; now the global bias is positive - there is a positive current.

A quantum random walk on a one-dimensional lattice involves a "quantum coin", a qubit - a quantum system with a two dimensional Hilbert space. Its state is a superposition $a|\downarrow\rangle+b|\uparrow\rangle$ with $|a|^{2}+|b|^{2}=1$, where $|\downarrow\rangle$ and $|\uparrow\rangle$ are the eigenstates of $\sigma_{z}=\left(\begin{array}{cc}1 & 0 \\ 0 & -1\end{array}\right)$. An unbiased coin flip is represented by the unitary transition matrix $\left(\begin{array}{ll}1 & i \\ i & 1\end{array}\right) / \sqrt{2}$, which means that the amplitude for the coin to remain in the same $\sigma_{z}$ eigenstate is $1 / \sqrt{2}$, while it has amplitude $i / \sqrt{2}$ to change eigenstates.

Letting $|x\rangle \in \mathbb{C}^{\mathbb{Z}}$ denote capital, and identifying $|\downarrow\rangle$ and $|\uparrow\rangle$ with 'lose' and 'win', respectively, one timestep of the unbiased quantum random walk (or, equivalently, one play of the quantum version of game $A$ ) is the unitary transformation defined by linear extension from

$$
\begin{aligned}
& |x, \downarrow\rangle \mapsto \frac{1}{\sqrt{2}}(|x-1, \downarrow\rangle+i|x+1, \uparrow\rangle) \\
& |x, \uparrow\rangle \mapsto \frac{1}{\sqrt{2}}(i|x-1, \downarrow\rangle+|x+1, \uparrow\rangle) .
\end{aligned}
$$

The quantum version of repeated play of game $A$ is to iterate this transformation repeatedly, delaying measurement until the end of the repetition, from an initial state representing capital 0 . In this paper the 


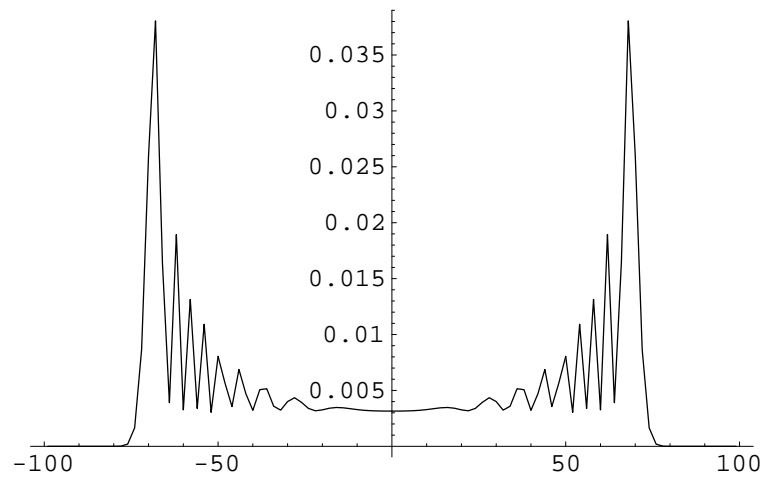

Figure 2. The payoff distribution for game $A$ after 100 plays. Since the distribution vanishes at $x \equiv T$ $\bmod 2$, a weighted average $(p(x-1)+2 p(x)+p(x+1)) / 4$ is plotted.

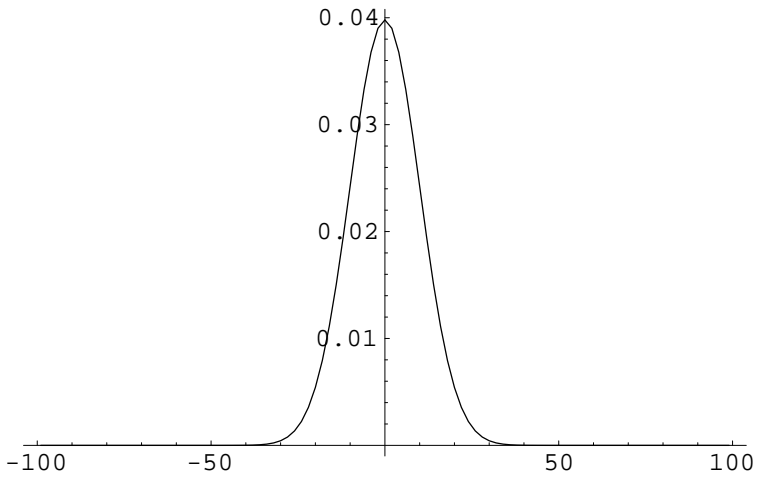

Figure 3. The payoff distribution for the classical game $A$ after 100 plays. This distribution has the same parity condition as does the quantum one, and is plotted the same way.

initial state is always $(|0, \downarrow\rangle+|0, \uparrow\rangle) / \sqrt{2}$. The outcome of a "position" measurement on a state $|\psi\rangle \in \mathbb{C}^{\mathbb{Z}} \otimes \mathbb{C}^{2}$ is drawn from a probability distribution with $\operatorname{Pr}(x)=|\langle\psi \mid x, \downarrow\rangle|^{2}+|\langle\psi \mid x, \uparrow\rangle|^{2}$. Figure 2 shows the probability distribution after 100 iterations of game $A$. Although this distribution looks nothing like the distribution of a classical randomly walking particle (shown in Figure 3 for a particle also initialized at $x=0$ ), it is symmetric, so the expected winnings $\langle x\rangle=\sum x \operatorname{Pr}(x)$ are always 0 for this unbiased quantum game.

Although we introduced it here as a repeated quantum game, the QLGA evolution rule (2.1) is a discretization of the evolution of a Dirac particle in $1+1$ dimensions $^{7}$, in which context the rule is described as "scattering" followed by "advection". This indicates how a bias should be introduced, given the goal of modelling a ratchet potential: In the QLGA model, potentials are implemented by $x$-dependent phase multiplication $^{11}$. Thus the quantum version of the $B$ game consists of the unbiased transition matrix, multiplied by a phase $e^{-i V(x)}$, where $V_{B}(x)=\beta\left(1-\frac{1}{2}(x \bmod 3)\right)$ is a sawtooth potential, followed by advection:

$$
\begin{aligned}
& |x, \downarrow\rangle \mapsto \frac{1}{\sqrt{2}} e^{-i V_{B}(x)}(|x-1, \downarrow\rangle+i|x+1, \uparrow\rangle) \\
& |x, \uparrow\rangle \mapsto \frac{1}{\sqrt{2}} e^{-i V_{B}(x)}(i|x-1, \downarrow\rangle+|x+1, \uparrow\rangle) .
\end{aligned}
$$

With this kind of modification of the evolution rule, the expectation value of $x$ is no longer constantly zero. Figure 4 suggests that for $\beta=-\pi / 3$, game $B$ is a fair game, in the same sense that the classical game $B$ is a fair game: The expected winnings are negative, but asymptotically constantthe gambler's losses do not increase in proportion to the number of times he plays game $B$. Furthermore, the current $\mathrm{d}\langle x\rangle / \mathrm{d} t \neq 0$, although it oscillates around zero.

\section{DISCRETE QUANTUM RATCHETS}

As we saw in Figure 1, alternating the classical games $A$ and $B$ mimics a flashing ratchet and from two fair games produces a winning game - in the strong sense that the gambler's winnings are proportional to the number of plays.

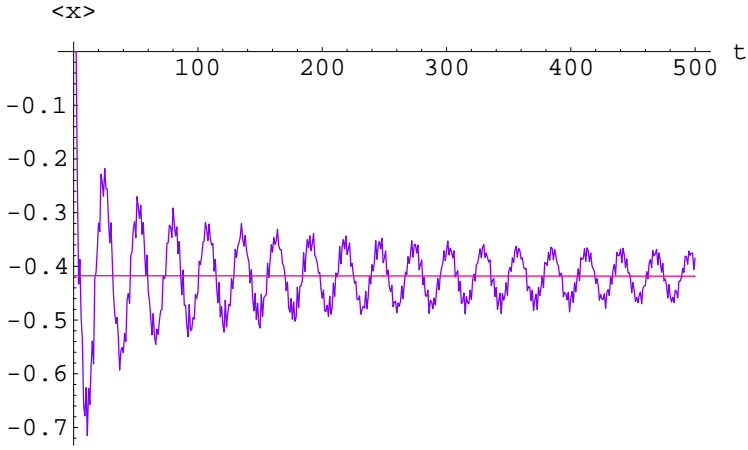

Figure 4. The expected payoffs for quantum game $B$, as a function of number of plays $t$. Like the results for classical game $B$ shown in Figure 1, after a (somewhat longer) initial oscillatory phase the payoffs converge to a constant (negative) value. Thus the quantum game $B$ is also fair. 


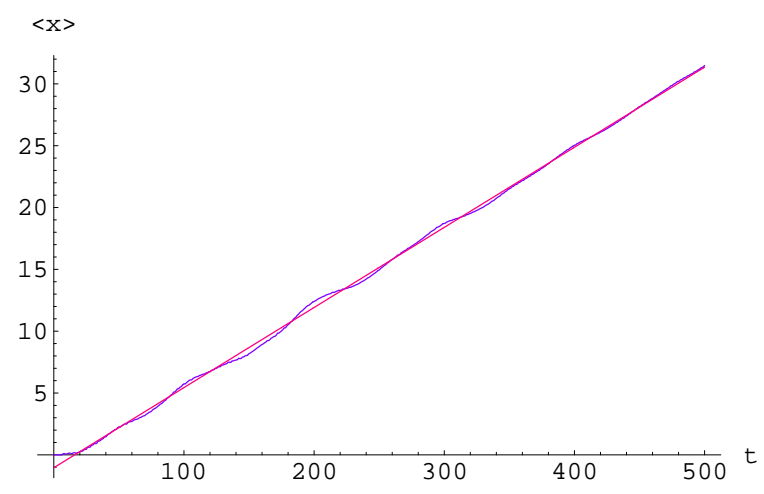

Figure 5. The expected payoffs for the repeating sequence $A A A A B$ of quantum games. Again, like the result for the sequence of classical games shown in Figure 1, this is a winning game. There is a positive current which can be approximated as the slope of the linear fit shown.

A similar result holds for the fair quantum games $A$ and $B$. Figure 5 shows that playing these games in the sequence $A A A A B$, repeatedly, produces a winning game, in the same strong sense. That is, asymptotically there is a positive current $\mathrm{d}\langle x\rangle / \mathrm{d} t \approx 0.0648$, where this numerical value is obtained by a least squares fit of a line to the sequence of values $\langle x(t)\rangle$.

Just as in the classical case, the amount of current depends on exactly which repeating sequence of games is played. For example, the sequence $A A A B B$, repeating, gives only a current of approximately 0.0357 . In fact, it also depends on the point at which the sequence begins: $A A A A B$ gives the same bi-infinite sequence as $B A A A A$, but the latter gives a completely different result starting from the initial state localized at $0-$ no ratcheting at all.* These results are shown in Figures 6 and 7. For the rest of this paper we will concentrate on the sequence $A A A A B$ that produces the relatively strong current shown in Figure 5; this case will demonstrate the most dramatic impact of the addition of noise to the model.

\section{ADDING NOISE}

As we noted in $\S 1$, experiments on ratcheting behavior in real systems unavoidably involve interaction with the environment, i.e., noise. We may expect this interaction to be particularly detrimental to quantum effects as these depend on delicate coherences between quantum amplitudes for different states. At the extreme where the environment (completely) measures the quantum system, all coherence is destroyed. If

* The results in papers [3] and [8] are for the sequence $A A A A B$, despite their description in those papers as coming from the sequence $B A A A A$. We thank Derek Abbott and Adrian Flitney for pointing out this mistake. 
the quantum random walks/QLGA described in $\S 2$ and $\S 3$ were completely measured at each timestep, the resulting random process would behave as a classical, unbiased random walk, independently of any potential imposed via (2.2).

Recent numerical and (approximate) analytical investigations of projective measurement of position ${ }^{12}$ and arbitrary positive operator valued measurement (POVM) of direction ${ }^{13}$ in quantum random walks have shown that any positive probability $p$ of measurement per timestep asymptotically destroys certain quantum features of the random walk. In particular, the variance of position is no longer proportional to the number of timesteps $T$, but rather to $\sqrt{T}$ as it is for the classical random walk. The transition occurs at $p T=O(1)$.

There is only a distant relation between these measurement models for the interaction of a quantum random walk with its environment and the more traditional models for interaction with a heat bath that have been applied to models for quantum ratchets ${ }^{5,6}$. The latter are based on the usual model of a heat bath as an infinite collection of harmonic oscillators coupled bilinearly to the particle position ${ }^{14,15}$. For this model the decoherence (or noise) in the system is a function of the temperature of the heat bath and the strength with which it is coupled to the quantum system. To construct a noisy quantum Parrondo game that can be compared to existing models for quantum ratchets, we need a discrete space and time model for interaction with such a bath of oscillators.

Since coherent quantum effects are the basis of successful quantum computations ${ }^{10}$, over the past several years there has been extensive investigation into decoherence - the effect of noise - of finite-dimensional quantum systems evolving discretely in time. The noise models developed for these systems apply directly to quantum random walks/QLGA and can be interpreted as exactly the sort of model for coupling to a heat bath that we want. Specifically, we consider the following error model: Impose periodic boundary conditions on the position variable $0 \leq x<N$. (This has no good interpretation in terms of a gambler's capital, so when we apply this model to define noisy quantum Parrondo games, the number of plays $T$ will be small enough $(T<4 N)$ that the periodicity in $x$ has no effect on the results.) An error basis for the Hilbert space $\mathbb{C}^{N}$ is given by $X^{i} Z^{j}, i, j \in \mathbb{Z}_{N}$, where $X$ and $Z$ are generalizations of the Pauli matrices that act on qubits:

$$
\begin{aligned}
X|x\rangle & =|x+1\rangle \\
Z|x\rangle & =\omega^{x}|x\rangle,
\end{aligned}
$$

where $\omega^{N}=1^{16}$. Since the interaction of a heat bath with particle position leads to a Gaussian error distribution with temperature-dependent width, the natural approximation in this discrete setting is a binomial distribution of the basis errors around $X^{0} Z^{0}$. That is, the error model for each timestep is

$$
\rho \mapsto \mathcal{E}(\rho)=(1-p) \rho+\frac{p}{6}\left(X \rho X^{\dagger}+X^{\dagger} \rho X+Z \rho Z^{\dagger}+Z^{\dagger} \rho Z+Y \rho Y^{\dagger}+Y^{\dagger} \rho Y\right),
$$

where $Y=Z X$ and $\rho$ is the density matrix for the quantum system. * The "probability of an error" $0 \leq p \leq 1$ combines the effects of the temperature of the heat bath representing the environment and the strength of the coupling constant to the quantum system.

\section{RESULTS}

Adding the noise (4.1) to game $A$, i.e., to an unbiased quantum random walk or a one-particle QLGA with constant potential, simply leads to a "smoothing" of the position distribution - an interpolation between the distributions of Figures 2 and 3. Similar results have been found for the measurement models of decoherence described in $\S 4$ that lead to $O(\sqrt{(} T))$ variance in position ${ }^{13,12}$. In all models, of courses, adding unbiased noise leaves the random walk unbiased, or the game fair. Our interest is in the effect of the noise (4.1) on the (strongly) winning game consisting of the sequence of plays $A A A A B$, repeated.

* We are abusing notation slightly in (4.1): The errors $X$, etc., only act on $\mathbb{C}^{N}$, while the Hilbert space here is $\mathbb{C}^{N} \otimes \mathbb{C}^{2}$. Thus we really mean $X \otimes I_{2}$, etc. 


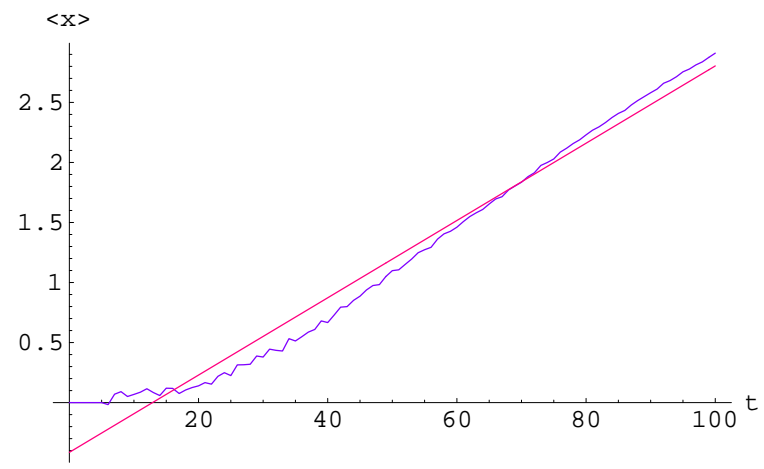

Figure 8. The expected payoffs for the repeating sequence $A A A A B$ of quantum games, with noise level $p=1 / 32$. On this timescale this is still a winning game, as it is for $p=0$.

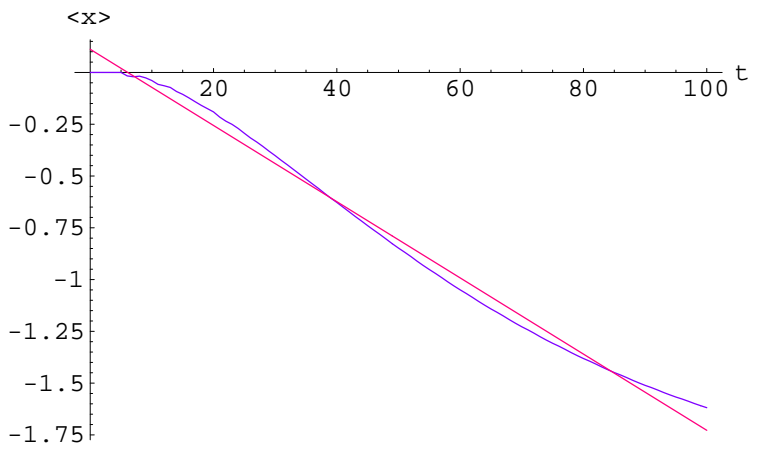

Figure 9. The expected payoffs for the repeating sequence $A A A A B$ of quantum games, with noise level $p=1 / 2$. On this timescale this is a losing game; the current has reversed.

As indicated by the definition of the error model (4.1) in terms of its effect on the density matrix of the quantum system, it is no longer sufficient to simply evolve a state vector. Let $U_{A}$ and $U_{B}$ be the unitary matrices representing the evolution rules (2.1) and (2.2), respectively. Then the evolution rule for our noisy quantum Parrondo game is

$$
\rho(t+1)=\left\{\begin{array}{ll}
\mathcal{E}\left(U_{A} \rho(t) U_{A}^{\dagger}\right) & t \not \equiv 4 \bmod 5 \\
\mathcal{E}\left(U_{B} \rho(t) U_{B}^{\dagger}\right) & t \equiv 4 \bmod 5
\end{array} .\right.
$$

Figure 8 shows the expected winnings for 100 iterations of this game when $p=\frac{1}{32}$ in (4.1). Although this is a relatively small error rate, it is larger than $1 / T$. Nevertheless, the resulting game is still (strongly) winning on this timescale, with a current of approximately 0.0322 , about half of the $p=0$ value.

For a sufficiently large error rate, e.g., $p=\frac{1}{2}$, however, the results are dramatically different. Figure 9 shows that in this case the expected winnings over 100 iterations are decreasing; the current is approximately -0.0184 . That is, with this much noise, the quantum $A A A A B$ game switches from being a winning game to being a losing game, at least on this timescale. This seems to be exactly the discrete analogue of the current inversions that occur in continuum quantum ratchets as the temperature of the heat bath changes ${ }^{5,6}$.

\section{CONCLUSIONS}

The unbiased quantum Parrondo games detailed in $\S 3$ can be interpreted as discrete unitary ratchets ${ }^{8}$. As such it is natural to investigate the influence of the environment on the behavior of this quantum system. In $\S 4$ we have presented an error model that is new in the context of quantum random walks ${ }^{13,12}$, but which is the discrete space and time version of interaction with a heat bath ${ }^{14,15}$. Simulations of the resulting noisy quantum Parrondo games display a phenomenon analogous to the current inversions that occur in continuum quantum ratchets - winning games are converted to losing ones as the noise level increases.

Since naïve simulations of noisy quantum Parrondo games are quadratically slower than simulations of unitary Parrondo games, the results shown in Figures 8 and 9 are for only 100 timesteps rather than the 500 timesteps shown in Figures 4-7. It would be interesting to analyze longer time behavior, preferably analytically, or at least with more sophisticated numerical methods. We are also in the process of exploring other directions in the parameter space of these games. Those results will be reported elsewhere. 


\section{ACKNOWLEDGEMENTS}

This work has been partially supported by the National Security Agency (NSA) and Advanced Research and Development Activity (ARDA) under Army Research Office (ARO) grant number DAAD19-01-1-0520 and by the Air Force Office of Scientific Research (AFOSR) under grant number F49620-01-1-0494.

\section{REFERENCES}

[1] J. M. R. Parrondo, "Parrondo's paradoxical games", http://seneca.fis.ucm.es/parr/GAMES.

[2] G. P. Harmer and D. Abbott, "Parrondo's paradox", Stat. Science 14 (1999) 206-213; "Losing strategies can win by Parrondo's paradox", Nature 402 (1999) 864.

[3] D. A. Meyer and H. Blumer, "Parrondo games as lattice gas automata", quant-ph/0110028; J. Statist. Phys. 107 (2002) 225-239.

[4] D. A. Meyer, "Quantum strategies", quant-ph/9804010; Phys. Rev. Lett. 82 (1999) 1052-1055.

[5] P. Reimann, M. Grifoni and P. Hänggi, "Quantum ratchets", Phys. Rev. Lett. 79 (1997) 10-13.

[6] S. Yukawa, M. Kikuchi, G. Tatara and H. Matsukawa, "Quantum ratchets", cond-mat/9706222; J. Phys. Soc. Jpn. 66 (1997) 2953-2956.

[7] D. A. Meyer, "From quantum cellular automata to quantum lattice gases", quant-ph/9604003; J. Statist. Phys. 85 (1996) 551-574.

[8] D. A. Meyer and H. Blumer, "Quantum Parrondo games: biased and unbiased", Fluctuation Noise Lett. 2 (2002) L257-L262.

[9] For a comprehensive review of the subject, see P. Reimann, "Brownian motors: noisy transport far from equilibrium", cond-mat/0010237; Phys. Rep. 361 (2002) 57-265.

[10] For a relatively current presentation of quantum computing, see M. A. Nielsen and I. L. Chuang, Quantum Computation and Quantum Information (Cambridge: Cambridge University Press 2000).

[11] D. A. Meyer, "Quantum mechanics of lattice gas automata: One particle plane waves and potentials", quant-ph/9611005; Phys. Rev. E 55 (1997) 5261-5269.

[12] V. Kendon and B. Tregenna, "Decoherence in discrete quantum walks", quant-ph/0301182.

[13] T. Brun, H. Carteret and A. Ambainis, "The quantum to classical transition for random walks", quantph/0208195; "Quantum random walks with decoherent coins", quant-ph/0210180; Phys. Rev. A 67 (2003) 032304/1-9.

[14] R. P. Feynman and F. L. Vernon, Jr., "The theory of a general quantum system interacting with a linear dissipative system", Ann. Phys. 24 (1963) 118-173.

[15] A. O. Caldeira and A. J. Leggett, "Quantum tunnelling in a dissipative system", Ann. Phys. 149 (1983) $374-456$.

[16] D. Gottesman, A. Kitaev and J. Preskill, "Encoding a qubit in an oscillator", Phys. Rev. A 64 012310/121. 